\title{
Planar and Rigid Pyrazine-Based TADF Emitter for Deep Blue Bright Organic Light-Emitting Diodes
}

\author{
Pachaiyappan Rajamalli ${ }^{+}{ }^{[a, c]}$ Dongyang Chen ${ }^{+}{ }^{[a]}$ Subeesh Madayanad Suresh, ${ }^{[a]}$ \\ Youichi Tsuchiya, ${ }^{[b]}$ Chihaya Adachi, ${ }^{*[b]}$ and Eli Zysman-Colman ${ }^{*[a]}$
}

Two blue thermally activated delayed fluorescence (TADF) emitters bearing di-tert-butyl carbazoles as the electron donor groups and pyrazine (DTCz-Pz) or dipyrazine (DTCz-Pz) as the electron acceptor are presented. The DFT calculations predict DTCz-Pz and DTCz-DPz to possess high $\mathrm{S}_{1}$ energies $(3.19 \mathrm{eV}$ and $3.08 \mathrm{eV}$, respectively), and relatively large $\Delta E_{S T}$ values ( $0.52 \mathrm{eV}$ and $0.56 \mathrm{eV}$, respectively). The closely layered intermediate triplet states between $S_{1}$ and $T_{1}$, predicted by DFT calculations, are expected to facilitate the reverse intersystem crossing (RISC) and improve spin-vibronic coupling efficiency between the excited states, even the relatively larger $\Delta \mathrm{E}_{\mathrm{ST}} \mathrm{s}$. The

\section{Introduction}

Recently, organic light-emitting diodes (OLEDs) using organic thermally activated delayed fluorescence (TADF) emitters have generated great interest as a cheaper alternative to phosphorescent OLEDs based on noble metal complexes. ${ }^{[1-3]}$ Triplet excitons can be converted to singlet excitons in TADF materials via reverse intersystem crossing (RISC) due to the presence of a very small singlet-triplet energy gap $\left(\Delta \mathrm{E}_{\mathrm{ST}}\right)$. This was realized by a molecular design that localizes the HOMO on a donor moiety and the LUMO on an acceptor moiety, usually incorporated within a highly twisted structure. Although a plethora of TADF emitters has been developed since the first examples of highefficiency TADF OLEDs in 2012, ${ }^{[1]}$ deep blue TADF OLEDs (CIE coordinates where $\mathrm{x}<0.15, \mathrm{y}<0.20$ ) remain underdeveloped, and their efficiencies and stabilities are still generally lower than sky blue and green TADF OLEDs. ${ }^{[4,5]}$ Therefore, the development

[a] Dr. P. Rajamalli, ${ }^{+}$D. Chen, ${ }^{+}$Dr. S. M. Suresh, Prof. E. Zysman-Colman Organic Semiconductor Centre, EaStCHEM School of Chemistry, University of St Andrews,

St Andrews, Fife, KY16 9ST, UK

E-mail: eli.zysman-colman@st-andrews.ac.uk

[b] Prof. Y. Tsuchiya, Prof. C. Adachi

Center for Organic Photonics and Electronics Research (OPERA), Kyushu University,

744 Motooka, Nishi-ku, Fukuoka 819-0395, Japan

E-mail: adachi@opera.kyushu-u.ac.jp

[c] Dr. P. Rajamalli ${ }^{+}$

Materials Research Centre, Indian Institute of Science,

CV Raman Rd, Bengalore-560012, Karnataka, India

$\left.{ }^{+}\right]$These authors contributed equally to this work.

Supporting information for this article is available on the WWW under https://doi.org/10.1002/ejoc.202100086

of (c) 2021 The Authors. European Journal of Organic Chemistry published by Wiley-VCH GmbH. This is an open access article under the terms of the Creative Commons Attribution License, which permits use, distribution and reproduction in any medium, provided the original work is properly cited.
$\Delta \mathrm{E}_{\mathrm{ST}} \mathrm{S}$ for DTCz-Pz and DTCz-DPz are $0.27 \mathrm{eV}$ and $0.38 \mathrm{eV}$, and both molecules show high photoluminescence quantum yields ( $65 \%$, and $70 \%$, respectively). The decay lifetimes show temperature dependence in a PPT host, which is consistent with the fact that both molecules are TADF emitters in PPT. The OLEDs based on DTCz-Pz exhibit deep blue emission with $\lambda_{\mathrm{EL}}$ of $460 \mathrm{~nm}$ and $\mathrm{CIE}$ of $(0.15,0.16)$. The maximum external quantum efficiency $\left(E E_{\max }\right)$ reaches $11.6 \%$, with a maximum luminance $\left(L_{\text {max }}\right)$ of up to $6892 \mathrm{cdm}^{-2}$, while the device based on DTCzDPz exhibits sky blue emission with $\lambda_{\mathrm{EL}}$ of $484 \mathrm{~nm}$ and CIE of $(0.15,0.30)$, an $E Q E_{\max }$ of $7.2 \%$, and $L_{\max }$ of $8802 \mathrm{~cd} \mathrm{~m}^{-2}$.

of alternative, highly efficient blue-emitting materials remains highly desired. Sensible molecular structure design as well as electron donor and acceptor selection are required in order to obtain deep blue emission.

The nitrogen-containing heterocyclic acceptor triazine has been used widely in blue TADF emitters due to its high triplet energy (3.27 eV in 3-methylpentane ${ }^{[6]}$ ) and relatively weak electron-accepting properties $\left(E_{\text {red }}^{1 / 2}=-2.1 \mathrm{~V}\right.$ vs SCE in $\left.\mathrm{MeCN}^{[7]}\right){ }^{[8-10]}$ A prototypical example, DMAC-TRZ, contains a 2,4,6-triphenyl-1,3,5-triazine (TRZ) as the electron-acceptor and 9,9-dimethyl-9,10-dihydroacridine (DMAC) as the electron donor. This compound is a sky-blue emitter where the OLED showed $\lambda_{\mathrm{EL}}=504 \mathrm{~nm}$ and $\mathrm{CIE}$ coordinates of $(0.22,0.50)$ in $\mathrm{mCPCN}$ and, along with a high maximum external quantum efficiency $\left(E Q E_{\text {max }}\right)$ of $26.5 \%$. Researchers have tried to tune the emission color further towards the blue through structure optimization (Figure 1). ${ }^{[1]}$ Kaji et al. aimed decreasing the electron affinity of the electron acceptor by replacing the nonbridging phenyl rings in TRZ with adamantyl groups. The molecule MA-TA exhibited a destabilized LUMO of $-2.96 \mathrm{eV}$ compared to $-3.12 \mathrm{eV}$ for DMAC-TRZ, and a correspondingly destabilized $S_{1}$ level of $2.90 \mathrm{eV}$ (in toluene) compared to $2.74 \mathrm{eV}$ (in toluene) for DMAC-TRZ. ${ }^{[9,11]}$ As a result, the OLED using MATA exhibited deep-blue emission with $\lambda_{\mathrm{EL}}=465 \mathrm{~nm}$ and $\mathrm{CIE}$ coordinates of $(0.15,0.16)$, along with a high $\mathrm{EQE}_{\max }$ of $22.1 \% .{ }^{[9]}$ Adachi et al. further pushed the color towards the deep-blue region by using a weaker electron donor in 3,6-dimethylcarbazole coupled with a methylphenylene bridge that, due to the presence of the methyl group, increasing the torsion angle of the donor, leading to reduced conjugation, smaller $\Delta \mathrm{E}_{\mathrm{ST}}$ and a higher $\mathrm{S}_{1}$ energy. ${ }^{\left[{ }^{[8]}\right.}$ The molecule $\mathrm{CZ}-\mathrm{TRZ3}$ possesses a shallow LUMO of $-2.71 \mathrm{eV}$ (in DCM) and a high $\mathrm{S}_{1}$ energy of $3.10 \mathrm{eV}$ (in toluene). ${ }^{[8]}$ The OLEDs based on Cz-TRZ3 exhibited deep-blue emission with $\mathrm{CIE}$ coordinates $(0.15,0.10)$ and $\mathrm{EQE}_{\max }$ 
of $19.2 \%{ }^{[8]}$ On the other hand, Chou et al. found that by simply replacing the triazine with pyrimidine can push the emission to higher energy as well. ${ }^{[12]}$ The molecule T3 possesses a much destabilized LUMO energy of $-2.37 \mathrm{eV}$ (in $\mathrm{MeCN}$ ) and an $\mathrm{S}_{1}$ energy of $2.95 \mathrm{eV}$ (in toluene), while the device based on T3 exhibited blue emission with $\lambda_{\mathrm{EL}}=465 \mathrm{~nm}$ and CIE coordinates

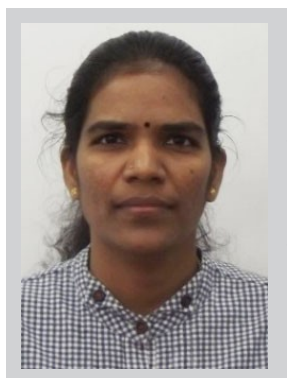

P. Rajamalli obtained her Ph.D. from the Indian Institute of Technology Madras under the guidance of Prof. Edaman Prasad, where she focused on developing dendrimer-derived luminescent materials. After completing her Ph.D. in 2012, she received a NSC postdoctoral fellowship and worked with Prof. Chien-Hong Cheng at National Tsing Hua University. In 2016, she joined the Zysman-Colman research group at the University of St Andrews, and in 2017 she received a prestigious Marie Skłodowska-Curie Individual Fellowship. During her post-doctoral fellowships, she has focused on the development of thermally activated delayed fluorescence emitters for organic lightemitting diodes. In 2019, she joined as an Assistant Professor at the Materials Research Centre, Indian Institute of Science. Currently, her research mainly focusses on the development of organic material for OLED applications.

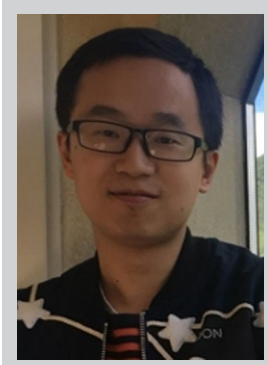

Dongyang Chen comes from Hunan province, China. He obtained his Bachelor of Science degree from Beijing Institute of Technology and his Master's degree from the Chinese Academy of Science. He joined the ZysmanColman group in St Andrews in 2016 as Ph.D. student and he works on TADF emitters design.

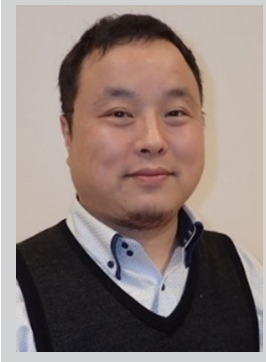

Youichi Tsuchiya is a research fellow specialappointment as an associate professor of Kyushu University's Center for Organic Photonics and Electronics Research (OPERA). He obtained his doctorate in Engineering in Material Science in 2004 from Nara Institute of Science and Technology (NAIST) and held positions as research fellow at National Institute of Advanced Industrial Science and Technology (AIST), Riken, and Institute of Systems, Information Technologies and nanotechnologies (ISIT) before working at Kyushu University. $\mathrm{He}$ had contributed to success of many research project with his research field related to functional materials based on organic chemistry, physical chemistry and supramolecular chemistry.

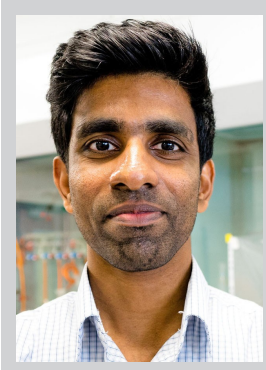

Subeesh Madayanad Suresh obtained his M.Sc. in Analytical Chemistry from Maharajas College Ernakulum. He then moved to Pusan National University in 2017 for a Ph.D. under the supervision of Prof. Youngson Choe. During this period, his research was focused on developing small organic molecules for light-emitting electrochemical cells (LEECs). He then moved to the University of St Andrews for a post-doctoral research with Prof. Eli Zysman-Colman on thermally activated delayed fluorescence (TADF) emitters for organic light-emitting diodes (OLEDs). In 2019, he was awarded with a
Marie Skłodowska-Curie Individual Fellowship with Prof. Zysman-Colman for developing narrow band emitters for OLEDs and LEECs.

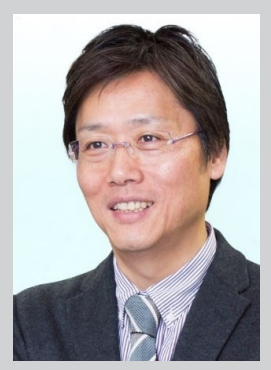

Chihaya Adachi is a distinguished professor at Kyushu University and director of Kyushu University's Center for Organic Photonics and Electronics Research (OPERA). Chihaya Adachi obtained his doctorate in Materials Science and Technology in 1991 from Kyushu University and held positions as at the Chemical Products R\&D Center at Ricoh Co., the Department of Functional Polymer Science at Shinshu University, the Department of Electrical Engineering at Princeton University, and Chitose Institute of Science and Technology before returning to Kyushu University as a professor.

Adachi's research combines the areas of chemistry, physics, and electronics to advance the field of organic light-emitting materials and devices from both the materials and device perspectives through the design of new molecules with novel properties, the study of processes occurring in individual materials and complete devices, and the exploration of new device structures, and he has co-authored over 600 research papers.

Recent awards he has received include a 2019 the $24^{\text {th }}$ Nagoya of Organic Chemistry "the silver medal ", a 2017 Nishina Memorial Prize and a 2016 Thomson Reuters Research Front Award, and he was named a 2018-2019 Highly Cited Researcher for the Cross-Field category.

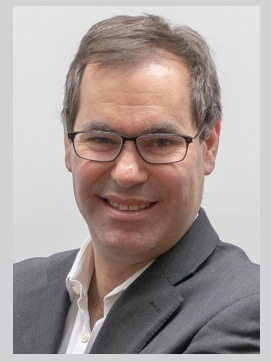

Eli Zysman-Colman obtained his Ph.D. from McGill University in 2003 under the supervision of Prof. David N. Harpp as an FCAR scholar, conducting research in physical organic sulfur chemistry. He then completed two postdoctoral fellowships, one in supramolecular chemistry with Prof. Jay Siegel at the Organic Chemistry Institute, University of Zurich as an FQRNT fellow and the other in inorganic materials chemistry with Prof. Stefan Bernhard at Princeton University as a PCCM fellow. He joined the department of chemistry at the Université de Sherbrooke in Quebec, Canada as an assistant professor in 2007. In 2013, he moved to the University of St Andrews where he is presently Professor of Optoelectronic Materials, Fellow of the Royal Society of Chemistry and is the holder of a Royal Society Leverhulme Trust Senior Research Fellowship. His research program focuses on the rational design of: (I) luminophores for energy-efficient visual displays and flat panel lighting based on organic light emitting diode (OLED) and lightemitting electrochemical cell (LEEC) device architectures; (II) sensing materials employed in electrochemiluminescence; and (III) photoredox catalyst design for organic reactions. 


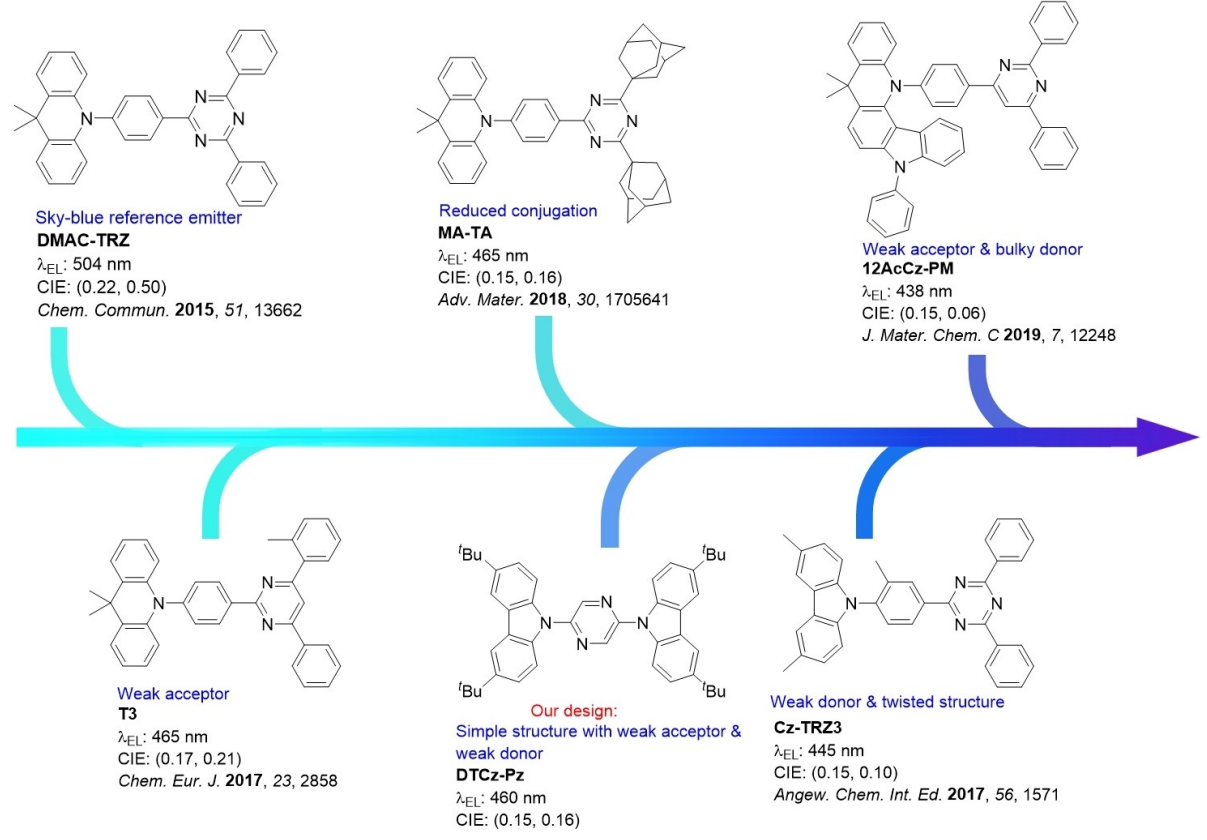

Figure 1. Chemical structures of heteroaromatic acceptor in blue TADF emitters design.

of $(0.17,0.21)$ and an $\mathrm{EQE}_{\max }$ of $11.8 \% .^{[12]}$ Wang et al. further blue-shifted the emission by using bespoke acridine-carbazole fused donor, and the bulky donor makes the molecule $12 \mathrm{AcCz}$ PM adopt a quasi-orthogonal conformation. ${ }^{[13]}$ The $S_{1}$ of $12 \mathrm{AcCz}-\mathrm{PM}$ is $3.06 \mathrm{eV}$ while the LUMO value is similar to T3 at $-2.31 \mathrm{eV}$ (in MeCN). ${ }^{[13]}$ The OLEDs based on 12AcCz-PM exhibited deep blue emission with $\lambda_{\mathrm{EL}}=438 \mathrm{~nm}$ and $\mathrm{CIE}$ coordinates $(0.15,0.06)$; however, the $\mathrm{EQE}_{\max }$ was only $5.7 \%$ due in part to the large $\Delta \mathrm{E}_{\mathrm{ST}}\left(0.39 \mathrm{eV}\right.$ in DPEPO). ${ }^{[13]}$

These examples in the literature have shown that triazine and pyrimidine acceptors are compatible with obtaining blue and deep blue TADF emitters and reasonably high efficiencies in OLEDs. The related N-heterocycle, pyrazine, has thus far not been explored for blue TADF emitter design, though the pyrazine has been used within an electron-acceptor design to tune its electron affinity and the corresponding energies of the emitters. ${ }^{[14-16]}$ Recently, Duan et al. reported a series of blue TADF emitters bearing pyrazine as an acceptor and benzofurocarbazoles or benzothieno-carbazoles as donor moieties. ${ }^{[17]}$ Amongst the molecules in the study, BFCZPZ2 possessed a moderately small $\Delta \mathrm{E}_{\mathrm{ST}}(0.31 \mathrm{eV}$ in PPT) and high photoluminescence quantum yield (PLQY) (91\% in PPT). ${ }^{[17]}$ The device based on BFCZPZ2 exhibited deep blue emission with $\lambda_{\mathrm{EL}}=464 \mathrm{~nm}$ and $\mathrm{CIE}$ coordinates of $(0.15,0.16)$, and the $\mathrm{EQE}_{\max }$ reached $21.3 \%$, but reduced to $5.1 \%$ at $10 \mathrm{~mA} / \mathrm{cm}^{2}{ }^{2[17]}$ This work showed the potential of pyrazine in the design of blue TADF materials.

In this work, we report two TADF emitters, 2,5-bis(3,6-di-tertbutyl-9H-carbazol-9-yl)pyrazine (DTCz-Pz) and 5,5'-bis(3,6-ditert-butyl-9H-carbazol-9-yl)-2,2'-bipyrazine (DTCz-DPz) (Figure 1). Both DTCz-Pz and DTCz-DPz possess a planar geometry showing high PLQY, $\Phi_{\mathrm{PL}}$, in doped thin films (70\% and $65 \%$ in PPT, respectively). Although the $\Delta E_{S T} S$ of DTCz-Pz and DTCz$\mathrm{DPz}$ are relatively large $(0.27 \mathrm{eV}$ and $0.38 \mathrm{eV}$ in PPT, respec- tively), the presence of an intermediate triplet state provides a viable route for TADF. The OLEDs based on DTCz-Pz and DTCzDPz exhibit deep blue and sky-blue emission with CIE coordinates of $(0.15,0.16)$ and $(0.15,0.30)$, and maximum EQE of $11.6 \%$ and $7.2 \%$, respectively.

\section{Results and Discussion}

\section{Theoretical Calculations}

The ground state $\left(\mathrm{S}_{0}\right)$ geometries of DTCz-Pz and DTCz-DPz were optimized by Density functional theory (DFT) and the excited states and their electronic configuration were predicted by time-dependent DFT calculations using the Tamm-Dancoff approximation (TDA-DFT) at the PBE0/6-31G(d,p) level of theory in the gas phase. ${ }^{[18,19]}$ The results are summarized in Figure 2. The DFT modeling predicts a rather flat conformation with average dihedral angles between the DTCz moieties and the pyrazine ring(s) of $\left[36.53(14)^{\circ}\right]$ and $\left[32.70(4)^{\circ}\right]$, for DTCz-Pz and DTCz-DPz, respectively. The relatively small dihedral angles align with those observed in the single crystal for a related TADF emitter, DTCz-DPzS $\left[38.20(8)^{\circ}\right]$, where the pyrazine ring was similarly connected to carbazole, indicating a likely intramolecular hydrogen bonding, analogously to that observed by us for related dipyrazinylsulfone TADF emitters. ${ }^{[14]}$ In both emitters, the HOMOs are distributed across the entire molecule while the LUMOs are mainly localized on the acceptor cores. The more strongly electron-accepting DPz in DTCz-DPz results in more stabilized HOMO and LUMO levels compared to those in DTC-Pz. Analogously, the $S_{1}$ and $T_{1}$ levels are predicted to be slightly stabilized in DTCz-DPz compared to DTCz-Pz. Both 


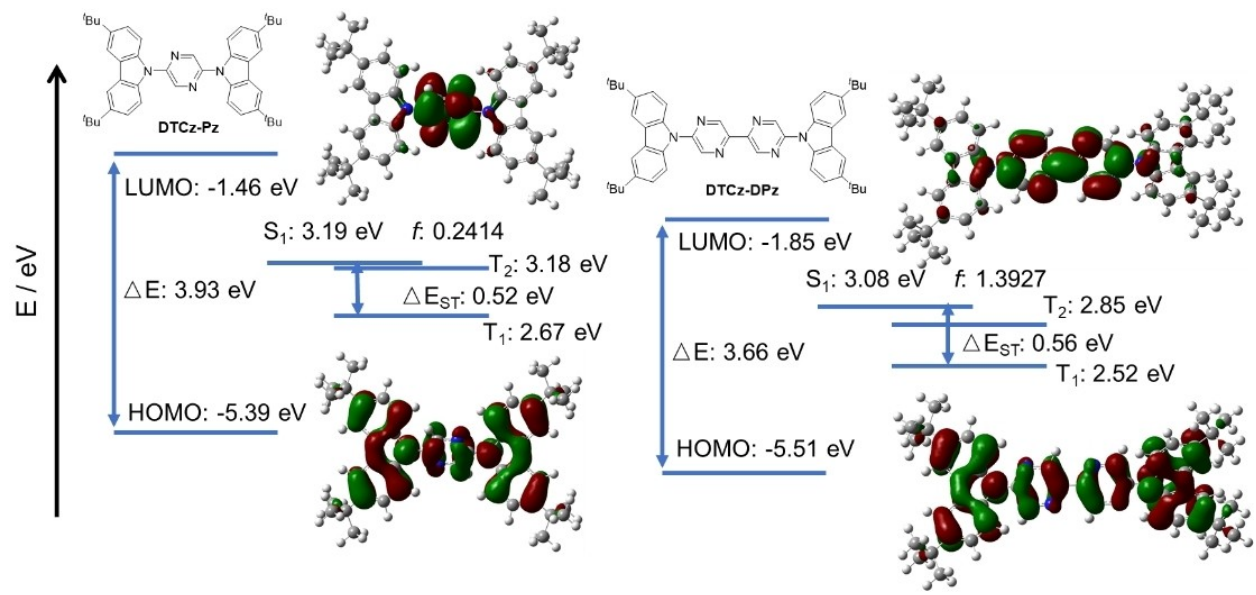

Figure 2. Theoretical modelling of the energies of the HOMO/LUMO orbitals and the $\mathrm{S}_{1}$ and $\mathrm{T}_{1}$ states of DTCz-Pz and DTCz-DPz in the gas phase and the electron density distribution of the frontier molecular orbitals (isovalue $=0.02$ ).

emitters show high oscillator strength $(0.24$ and 1.39 for DTCzPz and DTCz-DPz, respectively) and large $\Delta \mathrm{E}_{\mathrm{ST}}$ values $(0.52 \mathrm{eV}$ for DTCz-Pz and $0.56 \mathrm{eV}$ for DTCz-DPz). The higher energy intermediate triplet states of DTCz-Pz and DTCz-DPz were also studied (Figure S9). For DTCz-Pz, the $\mathrm{T}_{2}$ state $(3.18 \mathrm{eV})$ is nearly degenerate with the $S_{1}$ state $(3.19 \mathrm{eV})$. For DTCz-DPz, the $T_{2}$ state at $2.85 \mathrm{eV}$ results in a $\Delta \mathrm{E}_{\mathrm{S1T2} 2}$ of $0.23 \mathrm{eV}$. The nature of the $\mathrm{T}_{2}$ states for DTCz-Pz and DTCz-DPz is of locally excited (LE) character [HOMO to LUMO + 1 (85\%) for DTCz-Pz, HOMO-1 to LUMO + 1 (40\%) for DTCz-DPz] distributed over the whole molecule. This is different from the $\mathrm{S}_{1}$ and $\mathrm{T}_{1}$ states, which are dominated by a hybrid CT and LE transition from HOMO to LUMO. The presence of an intermediate $T_{2}$ state that is of different symmetry to $S_{1}$ in both DTCz-Pz and DTCz-DPz will contribute to a more efficient TADF as RISC will be faster, facilitated by spin-vibronic coupling between $T_{1}$ and $T_{2}$ and enhanced spin-orbit coupling (SOC) between $\mathrm{T}_{2}$ and $\mathrm{S}_{1} \cdot{ }^{[20,21]} \mathrm{We}$ calculated the $\left|V_{\text {Soc }}\right|^{2}$ values as the average spin-orbital coupling matrix elements (SOCME) between their $\mathrm{S}_{1} / \mathrm{T}_{1}$ states and $S_{1} / T_{2}$ states based on their optimized excited-state structures, ${ }^{[2]}$ which are shown in Table 1. The $\left|\mathrm{V}_{\mathrm{soc}}\right|^{2}$ value between $S_{1} / T_{1}$ for DTCz-Pz is $0.017 \mathrm{~cm}^{-2}$ and this increases to $0.517 \mathrm{~cm}^{-2}$ for $S_{1} / T_{2}$ due to the different orbital character of $T_{2}$ compared to $S_{1}$. Analogously, the $\left|V_{\text {soc }}\right|^{2}$ values for DTCz-DPz also increases from $0.003 \mathrm{~cm}^{-2}$ (between $S_{1} / T_{1}$ ) to $0.170 \mathrm{~cm}^{-2}$ (between $S_{1} / T_{2}$ ). The closely layered intermediate $T_{2}$ state and high SOCME values between the $S_{1} / T_{2}$ states provide an indirect route for RISC to occur despite the relatively large $\Delta E_{S T}$ for DTCz-Pz and DTCz-DPz.

\section{Synthesis}

DTCz-Pz was obtained in $77 \%$ yield via an Ullmann coupling reaction between two equivalents of 3,6-di-tert-butyl-9Hcarbazole (DTCz) and 2,5-dibromopyrazine. DTCz-DPz was obtained in moderate yield in a two-step protocol involving an Ullmann coupling between DTCz and 2-bromo-5-iodopyrazine followed by homocoupling of intermediate DTCzPzBr catalyzed by $\mathrm{Pd}\left(\mathrm{PPh}_{3}\right)_{4}$. (Scheme 1). Both emitters were characterized by combination of ${ }^{1} \mathrm{H}$ and ${ }^{13} \mathrm{C}$ NMR spectroscopy (Figures $\mathrm{S} 1-\mathrm{S} 4$ ), high-resolution mass spectrometry, melting point determination, and elemental analysis (Figures S5-56). The two emitters were purified by silica gel chromatography followed by temperature gradient vacuum sublimation, and the purity was verified by high performance liquid chromatography (HPLC) analysis (Figures S7-S8). The thermal properties of these emitters were determined by thermogravimetric analysis (Figure S13). The results show that these compounds possess very high $T_{\mathrm{d}}(5 \%$ weight loss) of $411^{\circ} \mathrm{C}$ and $459^{\circ} \mathrm{C}$, respectively for DTCz-Pz and DTzC-DPz.

\section{Electrochemistry}

Cyclic voltammetry (CV) and differential pulse voltammetry (DPV) measurements of DTCz-Pz and DTCz-DPz were carried out in dichloromethane (DCM) to estimate the HOMO and LUMO energies (Figure 3a). The CV trace of DTCz-Pz shows a pseudo-reversible oxidation wave with $\mathrm{E}_{\mathrm{ox}}=1.22 \mathrm{~V}$ (obtained

\begin{tabular}{|c|c|c|c|c|c|c|c|}
\hline Compound & $\mathrm{S}_{1}[\mathrm{eV}]$ & $\mathrm{T}_{1}[\mathrm{eV}]$ & $\Delta \mathrm{E}_{\mathrm{ST}}[\mathrm{eV}]$ & $\left|V_{S O C}^{S_{1} / T_{1}}\right|^{2}\left[\mathrm{~cm}^{-2}\right]$ & $\mathrm{T}_{2}[\mathrm{eV}]$ & $\Delta \mathrm{E}_{\mathrm{S1T2}}[\mathrm{eV}]$ & $\left|V_{\text {SOC }}^{S_{1} / T_{2}}\right|^{2}\left[\mathrm{~cm}^{-2}\right]$ \\
\hline DTCz-Pz & 3.19 & 2.67 & 0.52 & 0.017 & 3.18 & 0.01 & 0.517 \\
\hline DTCz-DPz & 3.08 & 2.52 & 0.56 & 0.003 & 2.85 & 0.23 & 0.170 \\
\hline
\end{tabular}



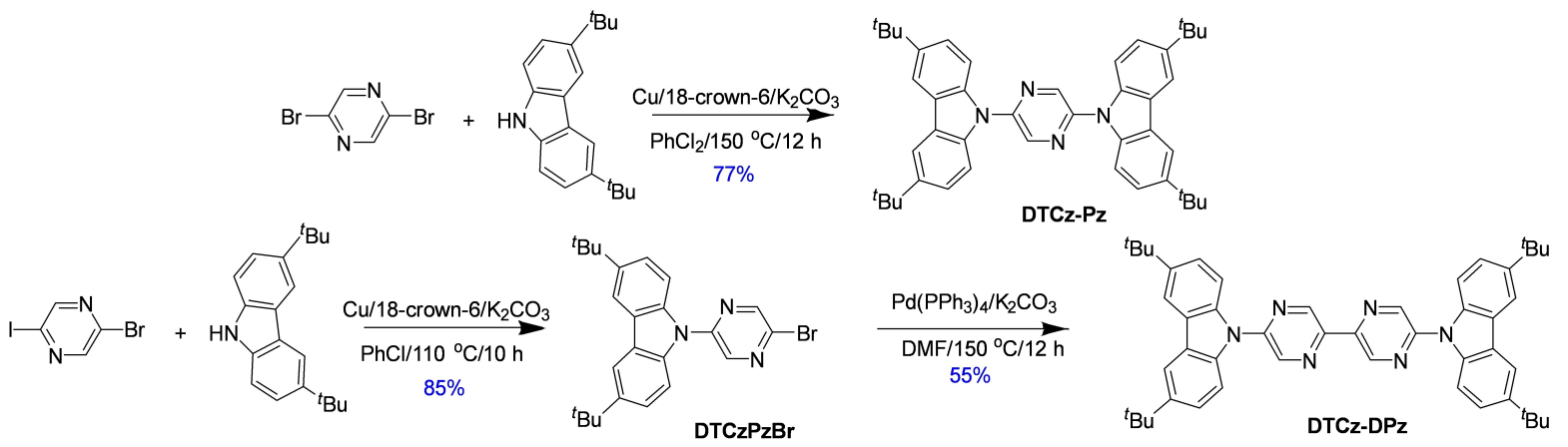

Scheme 1. Synthesis route of DTCz-Pz and DTCz-DPz.
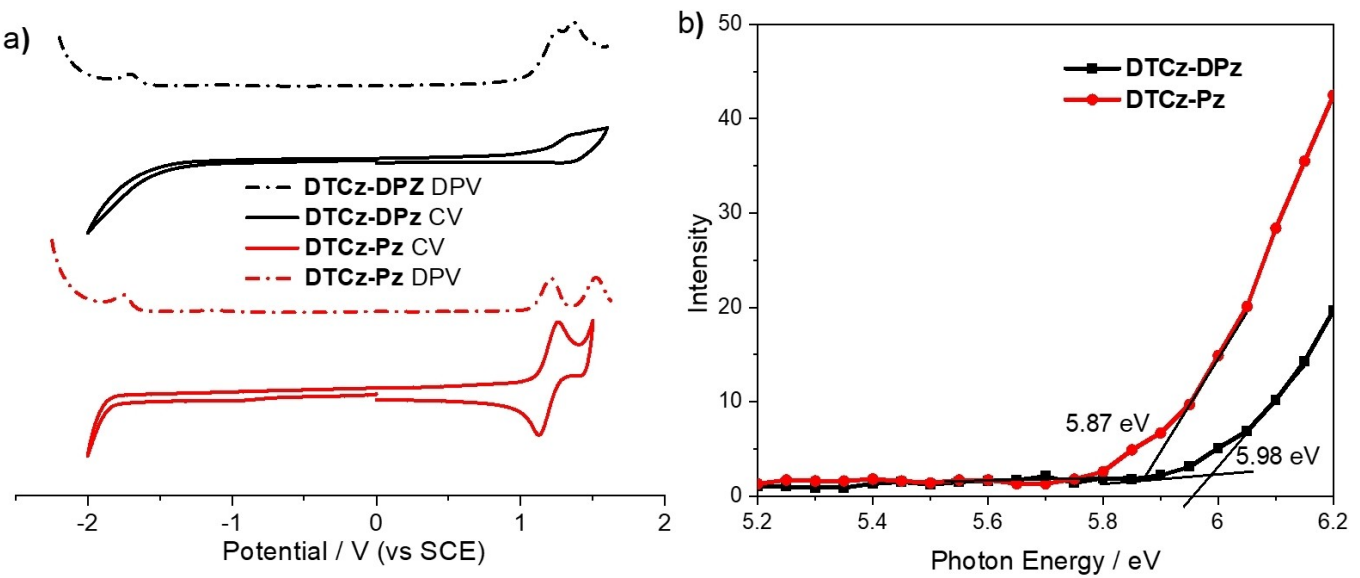

Figure 3. a) Cyclic Voltammograms (CV, solid lines) and Differential Pulse Voltammograms (DPV, dashed lines) for DTCz-Pz and DTCz-DPz in degassed DCM solution containing $\left[n \mathrm{nu}_{4} \mathrm{~N}\right] \mathrm{PF}_{6}$ as the supporting electrolyte and using $\mathrm{Fc} / \mathrm{Fc}^{+}$as an external standard $\left(\mathrm{Fc} / \mathrm{Fc}^{+}=0.46 \mathrm{eV}\right.$ versus $\mathrm{SCE}{ }^{[23]}$, scan rate $\left.=100 \mathrm{mVs}{ }^{-1}\right)$. b) Photoemission yield spectra of DTCz-Pz and DTCz-DPz.

from DPV) while DTCz-DPz exhibited an irreversible oxidation with $E_{o x}=1.23 \mathrm{~V}$ (obtained from DPV). No reduction wave was observed for either compound within the DCM solvent window. The corresponding HOMO levels of DTCz-Pz and DTCz-DPz are $-5.56 \mathrm{eV}$ and $-5.57 \mathrm{eV}$. Photoemission yield spectroscopy in air was used to measure the HOMO levels of neat thin films of the two emitters (Figure $3 \mathrm{~b}$ ) with values of $-5.87 \mathrm{eV}$ and $-5.98 \mathrm{eV}$ for DTCz-Pz and DTCz-DPz, respectively, which also qualitatively match the trends obtained from the DFT calculations and the electrochemistry. The LUMO levels for DTCz-Pz $(-2.58 \mathrm{eV})$, and DTCz-DPz $(-2.73 \mathrm{eV})$ were inferred from the HOMO energies and the optical gap $\left(E_{g}\right)$, which itself was determined from the intersection of the normalized absorption and fluorescence spectra in toluene (2.98 and $2.84 \mathrm{eV}$, respectively, for DTCz-Pz and DTCz-DPz, Figure S14).

\section{Photophysical properties}

The UV-vis absorption spectra of both emitters in toluene are shown in Figure 4a. The profiles match closely to the calculated absorption spectra by TDA-DFT (Figure S10). The TDA-DFT calculations predict that both emitters should exhibit hybridized local and charge-transfer (HLCT) transitions from HOMO to LUMO at $380 \mathrm{~nm}$ for DTCz-Pz and $400 \mathrm{~nm}$ for DTCz-DPz. For DTCz-DPz, the molar absorptivity of this band is much higher than that of DTCz-Pz due to the larger HOMO/LUMO overlap, which aligns with the predicted high oscillator strength. The absorption bands at $330 \mathrm{~nm}$ and $300 \mathrm{~nm}$ for DTCz-Pz are assigned to locally excited (LE) $\pi \rightarrow \pi^{*}$ transitions on the whole molecule and carbazole moiety, respectively, by TDA-DFT calculation (Table S2). For DTCz-DPz, TDA-DFT predicted a higher energy HLCT transition on $340 \mathrm{~nm}$ and a LE transition on the carbazole moiety at $300 \mathrm{~nm}$ (Table S3). The oscillator strengths $(f)$ for DTCz-Pz and DTCz-DPz are calculated to be 0.2514 and 0.5255 , respectively, from the spectra, ${ }^{[24]}$ which are consistent with the trend observed from the TDADFT calculations (0.2414 and 1.3927, respectively). The steady-state PL spectra for DTCz-Pz and DTC-DPz are shown in Figure $4 b$ and Figure $4 c$, respectively. In toluene, DTCz-Pz shows structureless emission with $\lambda_{P L}$ of $441 \mathrm{~nm}$, suggesting emission from a CT state, while the profile for DTCz-DPz in toluene has a slightly red-shifted $\lambda_{\mathrm{PL}}$ of $455 \mathrm{~nm}$. In $n$-hexane, both compounds show structured emission, though this is more pronounced in DTCzDPz, indicating emission from an LE state. There is a modest positive solvatochromism observed for DTCz-Pz while the 

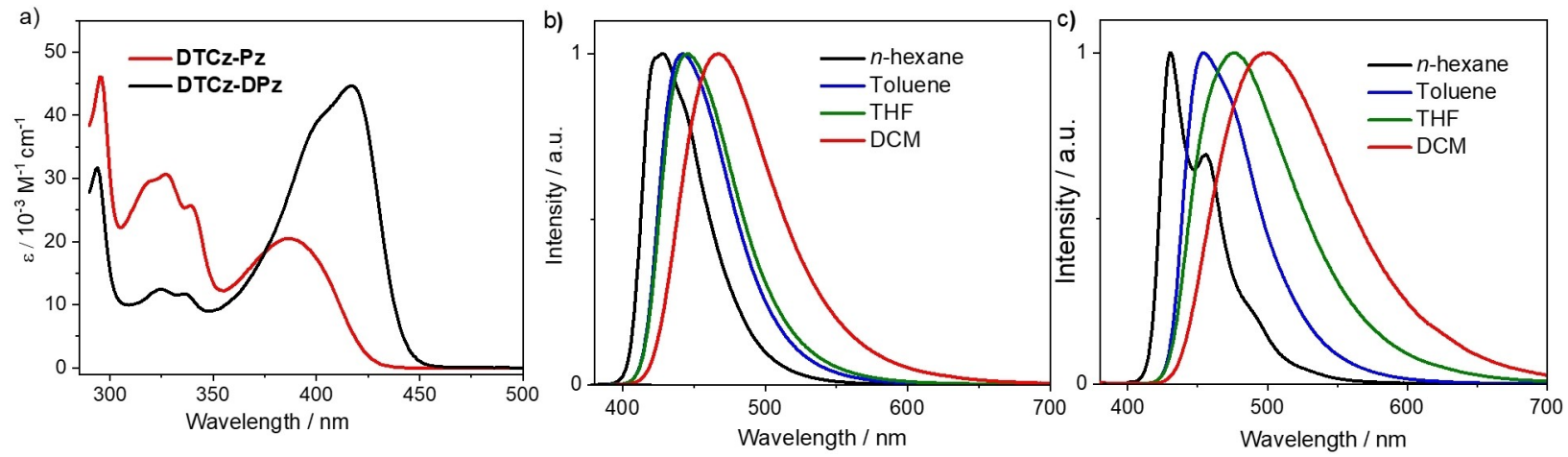

Figure 4. a) Absorption spectra of DTCz-Pz and DTCz-DPz in toluene; b) Solvatochromic PL study of DTCz-Pz; c) Solvatochromic PL study of DTCz-DPz $\left(\lambda_{\mathrm{exc}}=360 \mathrm{~nm}\right)$.

positive solvatochromism is more significant in DTCz-DPz, suggesting a more pronounced $\mathrm{CT}$ emissive state in this compound.

The time-resolved $\mathrm{PL}$ decays of these materials were measured in $10^{-5} \mathrm{M}$ toluene solution under nitrogen (Figure 5). In both compounds, the emission decays with bi-exponential kinetics. There is a nanosecond prompt emission, which occurs

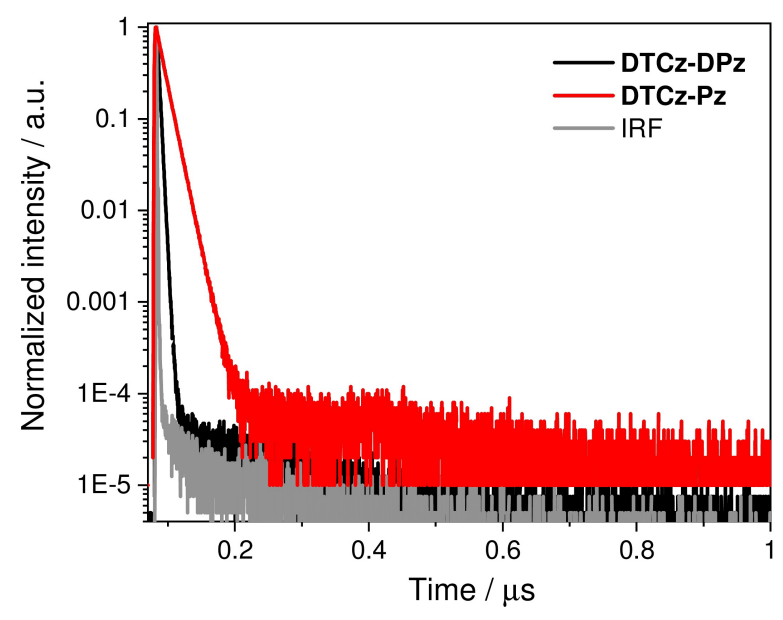

Figure 5. Transient PL decay of a) DTCz-Pz and b) DTCz-DPz in toluene $\left(\lambda_{\text {exc }}=378 \mathrm{~nm}\right)$ from direct radiative depopulation of the $S_{1}$ state with $\tau_{1}$ of $6.0 \mathrm{~ns}(63.4 \%)$ and $2.9 \mathrm{~ns}(85.7 \%)$ for DTCz-Pz and DTCz-DPz, respectively. There is a second component with a lifetime $\left(\tau_{2}\right)$ of 93 ns (36.6\%) and 121 ns (14.3\%) for DTCz-Pz and DTCz-DPz, respectively. Delayed emission is not observed for these compounds in solution.

We next investigated the photophysical properties of the two emitters in a high triplet energy host matrix, PPT $\left(\mathrm{E}_{\mathrm{T}}=\right.$ $2.95 \mathrm{eV}$ ) and the photophysical properties are summarized in Table 2. ${ }^{[25]}$ Thin films of each emitter doped at $7 \mathrm{wt} \%$ in PPT were prepared by vacuum deposition. DTCz-Pz shows structureless, broad emission for both prompt fluorescence and phosphorescence at $77 \mathrm{~K}$, pointing to ${ }^{1} \mathrm{CT}$ and ${ }^{3} \mathrm{CT}$ states (Figure 6). By contrast, DTCz-DPz shows broad emission for the prompt fluorescence spectrum and structured emission for the phosphorescence spectrum, indicating that for the latter, emission originates from a LE state on the DPz moiety. The calculated $\Delta E_{S T}$ values determined from the onsets of the prompt fluorescence and phosphorescence spectra are $0.27 \mathrm{eV}$ and $0.38 \mathrm{eV}$ for DTCz-Pz and DTCz-DPz, respectively.

The temperature-dependent time-resolved $\mathrm{PL}$ decays in doped PPT films are shown in Figure 7. The relative intensities of the delayed PL of both DTCz-Pz and DTCz-DPz decreased with decreasing temperature, which is the typical behavior for TADF emitters. Both compounds show a dominant prompt nanosecond fluorescence component and only a very small

\begin{tabular}{|c|c|c|c|c|c|c|}
\hline Compound & $\mathrm{S}_{1}[\mathrm{eV}]$ & $\mathrm{T}_{1}[\mathrm{eV}]$ & $\Delta \mathrm{E}_{\mathrm{ST}}[\mathrm{eV}]$ & $\Phi_{\mathrm{PL}}[\%]$ & $\tau_{\mathrm{p}}[\mathrm{ns}]$ & $\tau_{d}[\mathrm{~ms}]$ \\
\hline DTCz-Pz & 2.93 & 2.66 & 0.27 & 58 (air), $70\left(\mathrm{~N}_{2}\right)$ & 7.2 (77.7\%) 10.7 (22.3\%) & $\begin{array}{l}0.2(4.9 \%) \\
1.7(26.5 \%) \\
7.3(68.6 \%)\end{array}$ \\
\hline DTCz-DPz & 2.80 & 2.42 & 0.38 & 60 (air), $65\left(\mathrm{~N}_{2}\right)$ & 1.3 (16.9\%) 3.4 (71.7\%) 7.6 (11.3\%) & $\begin{array}{l}0.1(16.5 \%) \\
1.9(35.8 \%) \\
15.4(47.7 \%)\end{array}$ \\
\hline \multicolumn{7}{|c|}{$\begin{array}{l}\text { [a] } \mathrm{S}_{1}=\text { singlet state energy obtained from the onset of the prompt fluorescence spectra }(1-100 \mathrm{~ns}) \text { measured at } 77 \mathrm{~K} \text { with } \lambda_{\text {exc }}=343 \mathrm{~nm} ; \mathrm{T}_{1}=\text { triplet state } \\
\text { energy obtained from the onset of the phosphorescence spectra }(1-10 \mathrm{~ms}) \text { measured at } 77 \mathrm{~K} \mathrm{with} \lambda_{\text {exc }}=343 \mathrm{~nm} ; \Delta \mathrm{E}_{\mathrm{ST}}=\mathrm{E}\left(\mathrm{S}_{1}\right)-\mathrm{E}\left(\mathrm{T}_{1}\right) ; \Phi_{\mathrm{PL}}=\mathrm{photoluminescence} \\
\text { quantum yield measured using an integrating sphere under nitrogen flow with } \lambda_{\text {exc }}=340 \mathrm{~nm} ; \tau_{\mathrm{p}}=\text { prompt fluorescence lifetime measured at room } \\
\text { temperature with time window of } 100 \mathrm{~ns}\left(\lambda_{\text {exc }}=378 \mathrm{~nm}\right) . \tau_{\mathrm{d}}=\text { delayed fluorescence lifetime measured at room temperature with time window of } 1-40 \mathrm{~ms} \\
\left.\left(\lambda_{\text {exc }}=378 \mathrm{~nm}\right) \text {. All measurements were performed in co-doped PPT film ( } 7 \text { wt } \%\right) \text {. }\end{array}$} \\
\hline
\end{tabular}



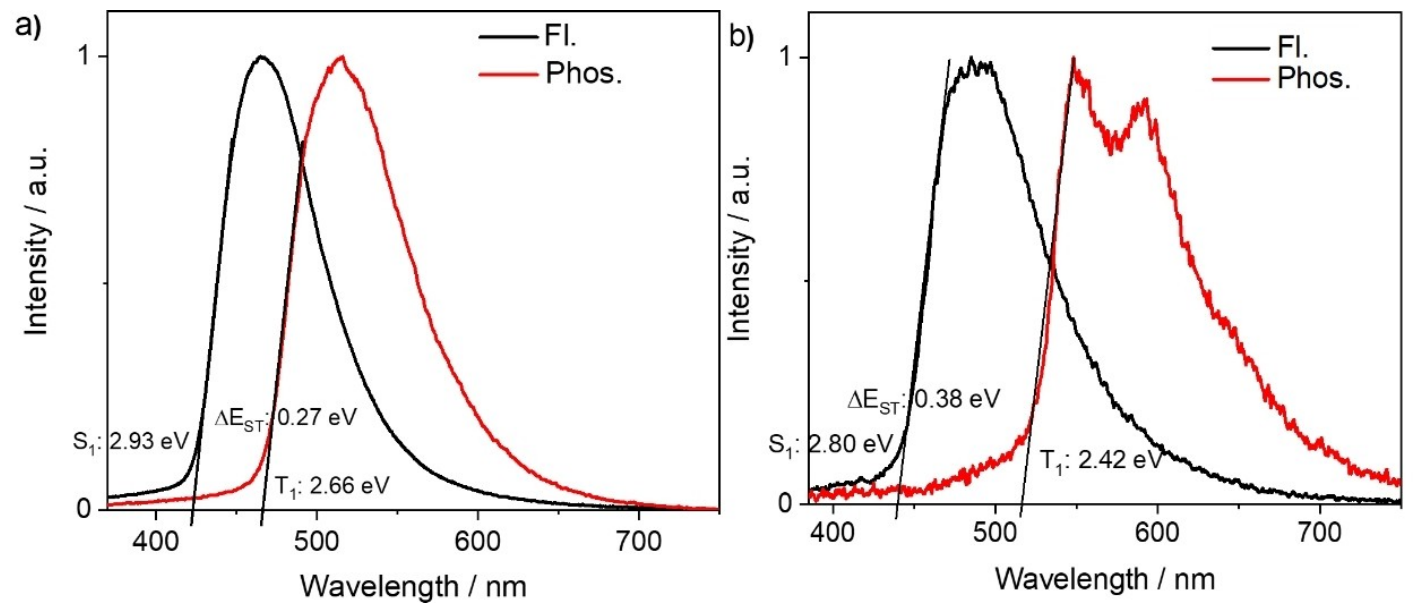

Figure 6. Prompt fluorescence (FI.) (1-100 ns) and phosphorescence (Phos.) (1-10 ms) spectra of a) DTCz-Pz and b) DTCz-DPz at $77 \mathrm{~K}$ as $7 \mathrm{wt} \%$ doped PPT films $\left(\lambda_{\text {exc }}=343 \mathrm{~nm}\right)$.
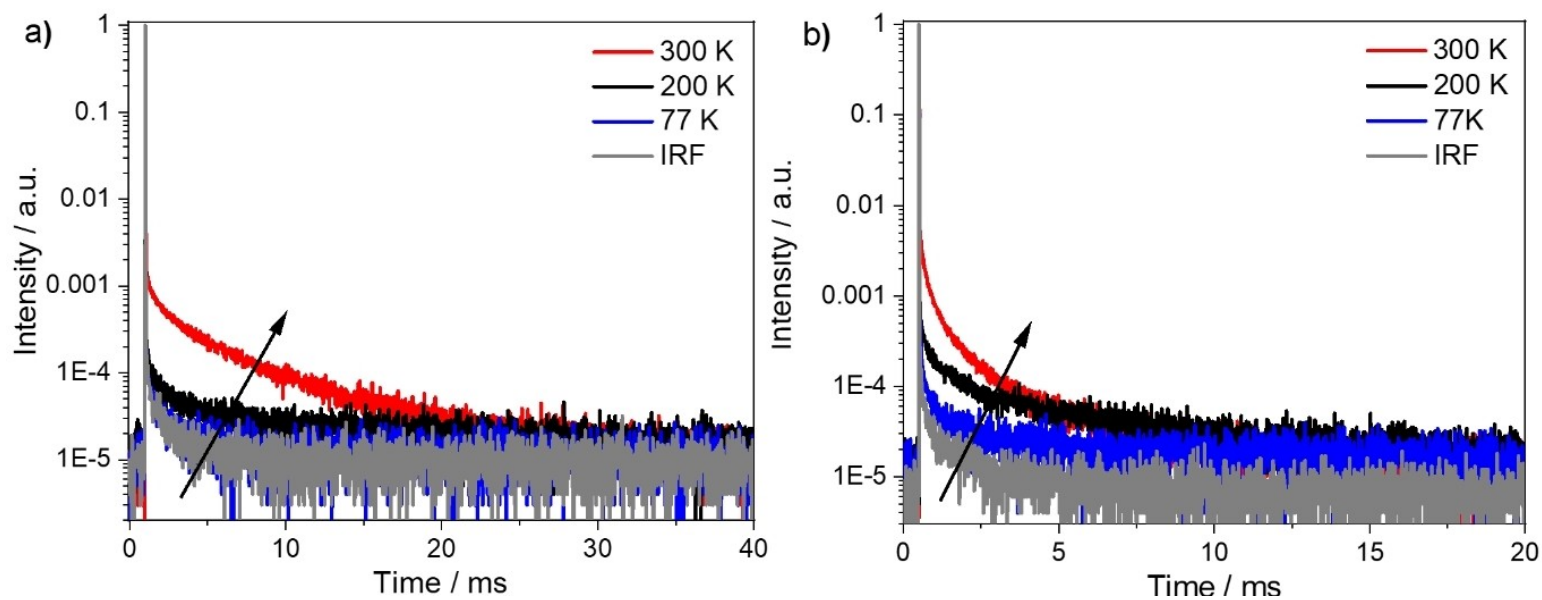

Figure 7. Normalized temperature-dependent transient PL decays of a) DTCz-Pz and b) DTCz-DPz in 7 wt $\%$ doped PPT films $\left(\lambda_{\text {exc }}=378 \mathrm{~nm}\right)$.

\begin{tabular}{|c|c|c|c|c|c|}
\hline Device & $\begin{array}{l}\text { EQE } \\
{[\%]}\end{array}$ & $\begin{array}{l}\mathrm{L}_{\max } \\
{\left[\mathrm{cd} / \mathrm{m}^{2}\right]}\end{array}$ & $\begin{array}{l}\mathrm{CE}_{\max } \\
{\left[\mathrm{cd} \mathrm{A} \mathrm{A}^{-1}\right]}\end{array}$ & $\begin{array}{l}\mathrm{PE}_{\max } \\
{\left[\operatorname{lm~W} W^{-1}\right]}\end{array}$ & $\begin{array}{l}\text { CIE (@8 V) } \\
\left(\lambda_{\mathrm{EL}}[\mathrm{nm}]\right)\end{array}$ \\
\hline DTCz-Pz & $11.6^{[a]} / 4.5^{[b]} / 2.5^{[c]}$ & 6892 & 13 & 11 & $\begin{array}{l}0.15,0.16 \\
(460)\end{array}$ \\
\hline DTCz-DPz & $7.2^{[\mathrm{a}]} / 3.8^{[\mathrm{b}]} / 2.8^{[\mathrm{c}]}$ & 8802 & 14 & 11 & $\begin{array}{l}0.15,0.30 \\
(484)\end{array}$ \\
\hline
\end{tabular}

delayed microsecond fluorescence component. For DTCz-Pz, the prompt fluorescence decays with bi-exponential kinetics with lifetimes of $7.2 \mathrm{~ns}(77.7 \%)$ and $10.7 \mathrm{~ns}(22.3 \%)$, while the delayed fluorescence decays according to triexponential kinetics with lifetimes of $0.2 \mathrm{~ms}(4.9 \%), 1.7 \mathrm{~ms}(26.5 \%), 7.3 \mathrm{~ms}$ (68.6\%). For DTCz-DPz, the prompt fluorescence lifetimes are $1.3 \mathrm{~ns}(16.9 \%), 3.4 \mathrm{~ns}(71.7 \%), 7.6 \mathrm{~ns}(11.3 \%)$ and the delayed fluorescence lifetimes are $0.1 \mathrm{~ms}(16.5 \%), 1.9 \mathrm{~ms}(35.8 \%)$, and $15.4 \mathrm{~ms}$ (47.7\%). The significantly longer delayed lifetimes in
DTCz-DPz are consistent with the larger $\Delta \mathrm{E}_{\mathrm{ST}}$ value. The delayed lifetimes are significantly reduced under air with $\tau_{d}$ of $4.2 \mu \mathrm{s}$ (99.1\%) for DTCz-Pz and $4.0 \mu$ s (99.5\%) for DTCz-DPz (Figure 8).

The absolute $\Phi_{\mathrm{PL}}$ values measured using an integrating sphere under argon and air for the toluene solutions, and co-doped films are summarized in Table 2. DTCz-DPz shows significantly higher $\Phi_{\mathrm{PL}}$ of $96 \%$ in degassed toluene than DTCz-Pz (69\%), while the $7 \mathrm{wt} \%$ PPT doped films are similarly bright with $\Phi_{\mathrm{PL}}$ of $70 \%$ and $65 \%$ for DTCz-Pz and DTCz-DPz, respectively. 

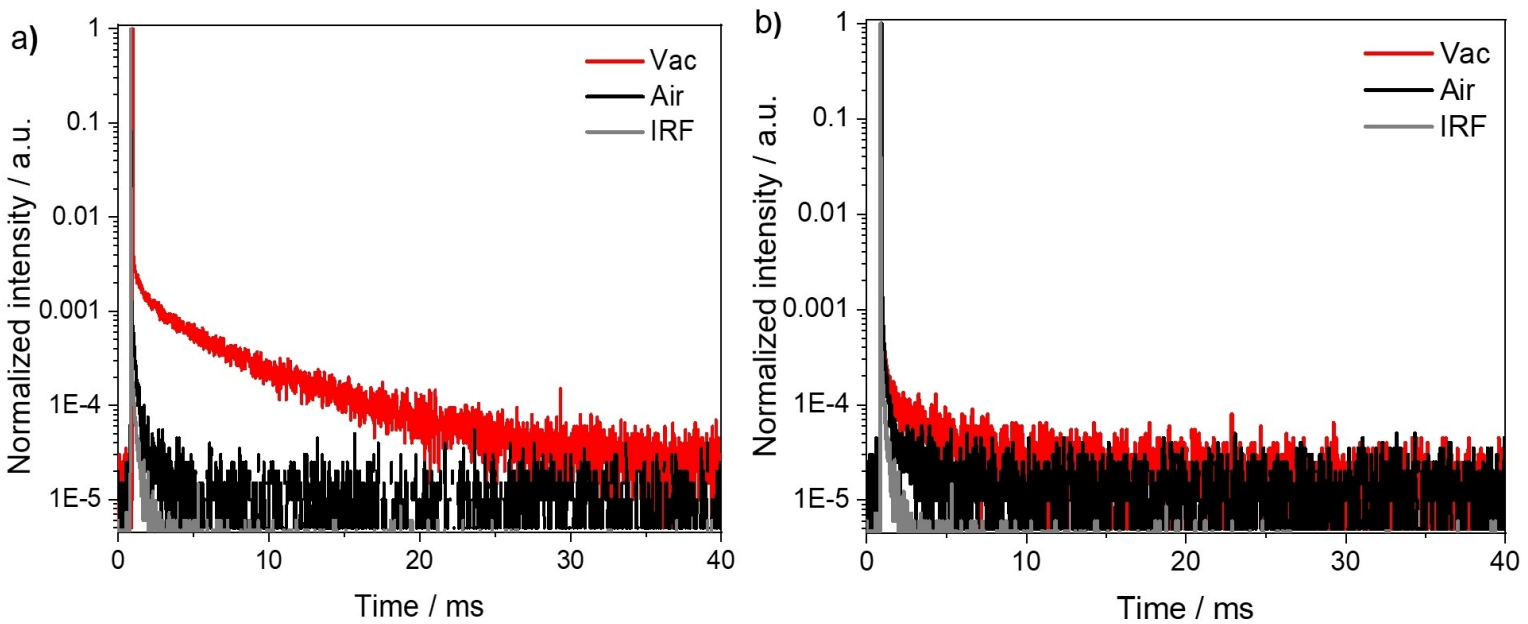

Figure 8. Transient PL decays of a) DTCz-Pz and b) DTCz-DPz co-doped at $7 \mathrm{wt} \%$ in PPT film, measured under vacuum and air $\left(\lambda_{\text {exc }}=378 \mathrm{~nm}\right)$.
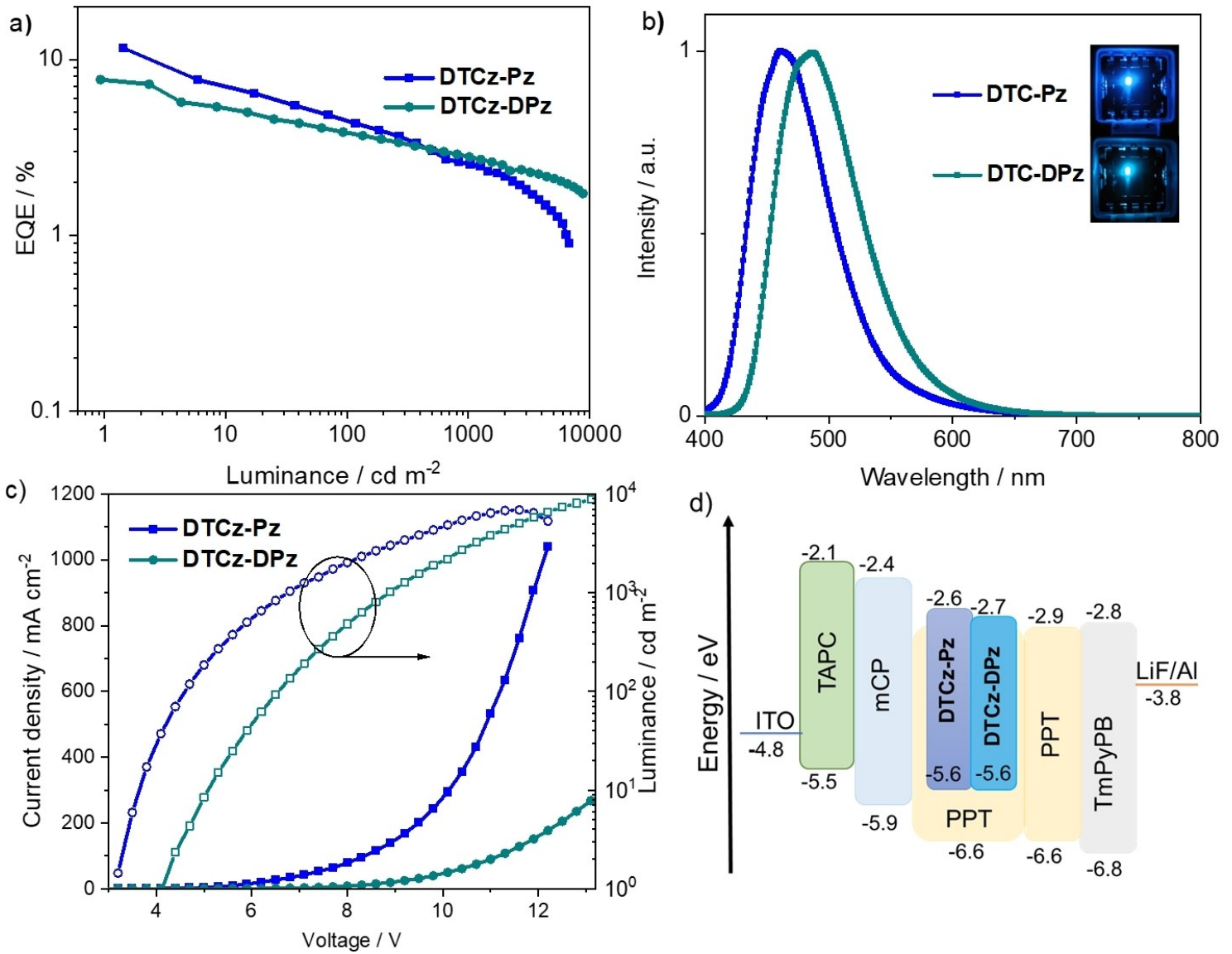

d)

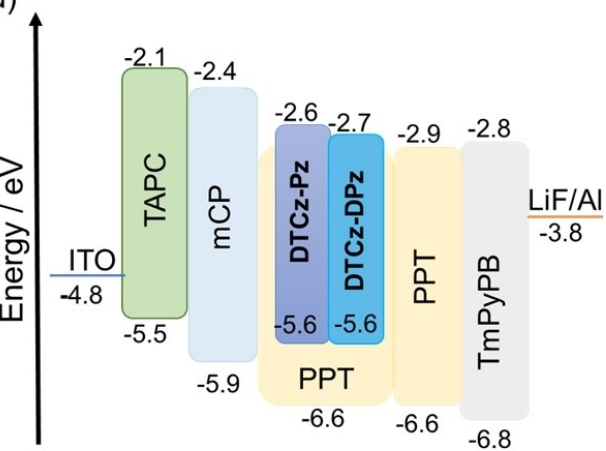

Figure 9. a) Luminance vs EQE, b) EL spectra of device (Inset: Photograph of device DTCz-Pz and DTCz-DPz, c) Current density-voltage-luminance characteristics, and d) Device structure: ITO/TAPC $(40 \mathrm{~nm}) / \mathrm{mCP}(10 \mathrm{~nm}) / \mathrm{PPT}:$ DTCz-Pz or DTCz-DPz $(7 \mathrm{wt} \%)(30 \mathrm{~nm}) / \mathrm{PPT}(10 \mathrm{~nm}) / \mathrm{TmPyPB}(30 \mathrm{~nm}) / \mathrm{LiF}(0.7 \mathrm{~nm}) /$ Al $(100 \mathrm{~nm})$.

\section{OLEDs}

Next, we investigated the electroluminescence (EL) properties of these two emitters. Multilayer devices were fabricated using these materials as dopants with the following structures: ITO/
TAPC $(40 \mathrm{~nm}) / \mathrm{mCP}(10 \mathrm{~nm}) / \mathrm{PPT}:$ DTCz-Pz or DTCz-DPz (7 wt\%) $(30 \mathrm{~nm}) / \mathrm{PPT}(10 \mathrm{~nm}) / \mathrm{TmPyPB}(30 \mathrm{~nm}) / \mathrm{LiF}(0.7 \mathrm{~nm}) / \mathrm{Al}(100 \mathrm{~nm})$. The performance of the devices is summarized in Figure 8 and Table 3. EQE-luminance curves reveal a maximum EQE (EQE $\left.E_{\max }\right)$ of $11.6 \%$ for the DTCz-Pz device and $7.2 \%$ for the DTCz-DPz 
device (Figure 9a). These devices show significant efficiency rolloff at high luminance. DTCz-Pz and DTCz-DPz-based devices show $\mathrm{EQE}_{100}$ of $4.5 \%$ and $3.8 \%$ at $100 \mathrm{~cd} / \mathrm{m}^{2}$ and $\mathrm{EQE}_{1000}$ is further reduced to $2.5 \%, 2.8 \%$ at $1000 \mathrm{~cd} / \mathrm{m}^{2}$, respectively. The EL spectra show the deep blue (for the DTCz-Pz device) and sky blue (for the DTCz-DPz device) emission with CIE coordinates of $(0.15,0.16)$ and $(0.15,0.30)$, respectively. High maximum luminance values of $6892 \mathrm{~cd} / \mathrm{m}^{2}$ and $8802 \mathrm{~cd} / \mathrm{m}^{2}$ were obtained for DTCz-Pz and DTCz-DPz devices, respectively.

\section{Conclusions}

We have synthesized two pyrazine-based emitters DTCz-Pz and DTCz-DPz bearing a mono and dipyrazine acceptors and di-tertbutyl carbazole as the donor group. These two materials show reasonably high photoluminescence quantum yields, ranging from 76 to $96 \%$, in both toluene solution and doped PPT thin films. The transient PL decay results in the doped thin film confirm that these compounds are TADF emitters. Blue-emitting OLEDs were fabricated using these emitting materials. The devices showed $\mathrm{EQE}_{\max }$ of $11.6 \%$ for the DTCz-Pz device with CIE $(0.15,0.16)$ and $7.2 \%$ for the DTCz-DPz device with CIE $(0.15,0.30)$.

\section{Supporting Information}

A previous version of this manuscript has been deposited on a preprint server (DOI: doi.org/10.26434/chemrxiv.13626668.v1). Instrumentation details, synthesis and chemical characterization (NMR spectra, elemental analysis reports, HPLC chromatograms), computational details are available in supporting information. The research data supporting this publication can be accessed at https://doi.org/10.17630/3008d05b-2053-435f80a8-91f7b544bf51.

\section{Acknowledgements}

We are grateful to the Engineering and Physical Sciences Research Council (EPSRC) for support from grants EP/P010482/1 and EP/ R035164/1, and Japan Society for the Promotion of Science (JSPS) Core-to-core Program. P. Rajamalli acknowledges support from a Marie Skłodowska-Curie Individual Fellowship (No. 749557). Subeesh M Suresh acknowledges support from a Marie Skłodowska-Curie Individual Fellowship (No. 838885). Dongyang Chen thanks the China Scholarship Council (201603780001). We thank for Dr. Tomas Matulaitis for help with temperature-dependent transient PL decays measurement.

\section{Conflict of Interest}

The authors declare no conflict of interest.

Keywords: Delayed fluorescence - Donor-acceptor • Organic light-emitting diodes $\cdot$ Pyrazine $\cdot$ TADF

[1] H. Uoyama, K. Goushi, K. Shizu, H. Nomura, C. Adachi, Nature 2012, 492, 234.

[2] P. Rajamalli, N. Senthilkumar, P. Y. Huang, C. C. Ren-Wu, H. W. Lin, C. H. Cheng, J. Am. Chem. Soc. 2017, 139, 10948.

[3] Z. Yang, Z. Mao, Z. Xie, Y. Zhang, S. Liu, J. Zhao, J. Xu, Z. Chi, M. P. Aldred, Chem. Soc. Rev. 2017, 46, 915.

[4] D. Zhang, M. Cai, Z. Bin, Y. Zhang, D. Zhang, L. Duan, Chem. Sci. 2016, 7, 3355.

[5] K. Suzuki, S. Kubo, K. Shizu, T. Fukushima, A. Wakamiya, Y. Murata, C. Adachi, H. Kaji, Angew. Chem. Int. Ed. 2015, 54, 15231.

[6] J. S. Beinen, J. G. Koben, W. G. Hodgson, J. Chem. Phys. 1966, 44, 3095.

[7] J. E. O'Reilly, P. J. Elving, J. Am. Chem. Soc. 1972, 94, 7941.

[8] L. S. Cui, H. Nomura, Y. Geng, J. U. k. Kim, H. Nakanotani, C. Adachi, Angew. Chem. Int. Ed. 2017, 56, 1571.

[9] Y. Wada, S. Kubo, H. Kaji, Adv. Mater. 2018, 30, 1.

[10] S. J. Woo, Y. Kim, S. K. Kwon, Y. H. Kim, J. J. Kim, ACS Appl. Mater. Interfaces 2019, 11, 7199.

[11] W.-L. Tsai, M.-H. Huang, W.-K. Lee, Y.-J. Hsu, K.-C. Pan, Y.-H. Huang, H.-C. Ting, M. Sarma, Y.-Y. Ho, H.-C. Hu, C.-C. Chen, M.-T. Lee, K.-T. Wong, C.-C. Wu, Chem. Commun. 2015, 51, 13662.

[12] P. Ganesan, R. Ranganathan, Y. Chi, X. K. Liu, C. S. Lee, S. H. Liu, G. H. Lee, T. C. Lin, Y. T. Chen, P. T. Chou, Chem. A Eur. J. 2017, 23, 2858.

[13] Q. Zhang, S. Sun, W. J. Chung, S. J. Yoon, Y. Wang, R. Guo, S. Ye, J. Y. Lee, L. Wang, J. Mater. Chem. C 2019, 7, 12248.

[14] P. L. Dos Santos, D. Chen, P. Rajamalli, T. Matulaitis, D. B. Cordes, A. M. Z. Slawin, D. Jacquemin, E. Zysman-Colman, I. D. W. Samuel, ACS Appl. Mater. Interfaces 2019, 11, 45171.

[15] S. Wang, Z. Cheng, X. Song, X. Yan, K. Ye, Y. Liu, G. Yang, Y. Wang, ACS Appl. Mater. Interfaces 2017, 9, 9892.

[16] J. Liu, K. Zhou, D. Wang, C. Deng, K. Duan, Q. Ai, Q. Zhang, Front. Chem. 2019, 7, 1.

[17] M. Cai, M. Auffray, D. Zhang, Y. Zhang, R. Nagata, Z. Lin, X. Tang, C. Chan, Y. Lee, T. Huang, X. Song, Y. Tsuchiya, C. Adachi, L. Duan, Chem. Eng. J. 2020, 127591.

[18] J. A. Pople, J. S. Binkley, R. Seeger, Int. J. Quantum Chem. 1976, $10,1$.

[19] C. Adamo, J. Chem. Phys. 1999, 110, 6158.

[20] M. K. Etherington, J. Gibson, H. F. Higginbotham, T. J. Penfold, A.P. Monkman, Nat. Commun. 2016, 7, 13680.

[21] L. Cui, A. J. Gillett, S. Zhang, H. Ye, Y. Liu, X. Chen, Z. Lin, E. W. Evans, W. K. Myers, T. K. Ronson, H. Nakanotani, S. Reineke, J. Bredas, C. Adachi, R. H. Friend, Nat. Photonics 2020, 14, 636.

[22] X. Gao, S. Bai, D. Fazzi, T. Niehaus, M. Barbatti, W. Thiel, J. Chem. Theory Comput. 2017, 13, 515.

[23] N. G. Connelly, W. E. Geiger, Chem. Rev. 1996, 96, 877.

[24] Y. Tsuchiya, K. Tsuji, K. Inada, F. Bencheikh, Y. Geng, H. S. Kwak, T. J. L. Mustard, M. D. Halls, H. Nakanotani, C. Adachi, Front. Chem. 2020, 8, 2.

[25] X. Cai, A. B. Padmaperuma, L. S. Sapochak, P. A. Vecchi, P. E. Burrows, Appl. Phys. Lett. 2008, 92, 083308.

Manuscript received: January 26, 2021

Revised manuscript received: February 17, 2021

Accepted manuscript online: February 19, 2021 


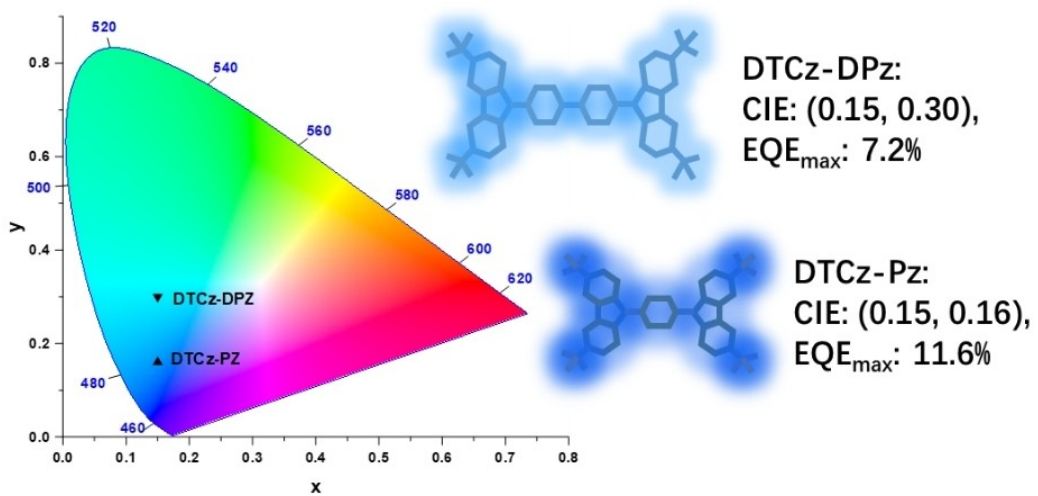

Dr. P. Rajamalli, D. Chen, Dr. S. M. Suresh, Prof. Y. Tsuchiya, Prof. C. Adachi*, Prof. E. Zysman-Colman*

$1-10$

Planar and Rigid Pyrazine-Based TADF Emitter for Deep Blue Bright Organic Light-Emitting Diodes

Two blue donor-acceptor thermally activated delayed fluorescence (TADF) emitters containing pyrazine or dipyrazine as electron-acceptor are presented. The OLEDs based on these two emitters exhibited blue and sky- blue emission with $\mathrm{CIE}$ of $(0.15,0.16)$ and $(0.15,0.30)$, respectively. The maximum external quantum efficiency for the blue device reached $11.6 \%$ while for sky blue device it is $7.2 \%$. 